\title{
SEQUENCING AND DETECTION OF POLYMORPHISMS IN THE 5' END OF THE HUMAN ENDOGENOUS RETROVIRAL ELEMENT, HRES-1
}

\author{
H.B. RASMUSSEN*, KNUD CHRISTENSEN ${ }^{\dagger}$, J. CLAUSEN* \\ *Institute of Life Sciences and Chemistry, Roskilde University, 4000 Roskilde, Denmark \\ †Dept. of Animal Genetics, Royal Veterinary and Agricultural Univ., 1870 Frederiksberg C, Denmark
}

\section{SUMMARY}

Fragments of the $5^{\prime}$ long terminal repeat (LTR) of the human endogenous retroviral element, HRES-1, were amplified. Single strand conformation analysis of these fragments in combination with sequencing revealed two polymorphic nucleotides, namely a HindIII and an Eco57I polymorphic site. Moreover, a number of differences from the previously published HRES-1 LTR sequence were detected. The linkage pattern of the two polymorphisms suggested the existence of three allelic forms of HRES-1. The frequencies of the genotypes and alleles of HRES-1 were determined in 158 individuals. The detection of HRES-1 markers may be useful to study associations between this endogenous retrovirus and various diseases.

KEY WORDS Endogenous Human retrovirus DNA polymorphisms

\section{INTRODUCTION}

The human genome contains a variety of endogenous retroviruses (Larsson et al., 1989). A considerable number of these endogenous proviruses share sequence homologies with murine and primate retroviruses; others are related to human exogenous retroviruses (Larsson et al., 1989). Increasing interest has been focused upon the endogenous retroviruses as factors potentially involved in carcinogenesis (Brodski et al., 1993, Sauter et al., 1995, Whartenby et al., 1993) and development of autoimmune diseases (Mountzand Talal, 1993) but many aspects concerning their possible functions remain unknown.

The human endogenous retroviral element HRES-1 shares sequence homologies with the human T cell leukemia viruses (HTLVs), human immunodeficiency virus type 2 (HIV-2) and feline sarcoma virus (Perl et al., 1989). This endogenous retroviral element is present in one copy or a very limited number of copies per haploid genome. The HRES1 locus has been assigned to chromosome 1 at q42 (Perl et al., 1991), a region which contains a fragile site (Sutherland et al., 1985).

So far, there is only sparse information with respect to the degree of individual variation in the composition of the human endogenous retroviruses. The present study was carried out to detect polymorphisms in HRES-1.

\section{MATERIALS AND METHODS}

Samples of DNA were isolated from peripheral blood leukocytes using digestion with proteinase- $\mathrm{K}$ (overnight at $55^{\circ} \mathrm{C}$ ) followed by phenol-chloroform-isoamyalcohol 
Table 1. HRES-1 oligonucleotides.

\begin{tabular}{llc}
\hline Designation & \multicolumn{1}{c}{ Sequence } & Nucleotide numbers \\
\hline A1 & CACTCCTTCAGAACTCTTGC & $196-215$ \\
A2 & CAGTCCCAGAGAGAGGCTCA & $456-475$ \\
B1 & GGACTAATTTGGCCTGAGGT & $421-440$ \\
B2 & TCTTTGTTTCCCCTGCAGCA & $692-711$ \\
C1 & AGTATTTGTCAAGCACCTCC & $665-684$ \\
C2 & TCCTGGTTTGGAATTCTTGC & $922-941$ \\
D1 & CCGTCACTGTAATCCCAATG & $898-917$ \\
D2 & TGCACAGCGCATTGCGTGATCC & $1134-1155$ \\
D3 & AAGCCAGCTTGATGTTCGGT & $1023-1042$ \\
& & \\
E1 & GGACAGATGGAGACAGGACT & $406-425$ \\
F1 & CTTGTCTCCTTCTTAAGAC & $756-774$ \\
\hline
\end{tabular}

Design of primers is based upon the previously published HRES-1 sequence (Perl et al., 1989). According to this sequence the two inverted repeats representing the boundaries of the 5' LTR, spans the sequences 309-313 and 990-993, respectively. Five different combinations of primers were used for the amplifications (A1-A2, B1-B2, C1-C2, D1-D2, D1-D3, A1-D2). Some of the primers were used for sequencing purposes (primer walking), including El and F1.

extraction. The blood samples were derived from 158 individuals, i.e. blood donors from Roskilde County Hospital and laboratory staff (all inhabitants of Denmark).

Primers for amplification and sequencing purposes are listed (Table 1). Amounts of $25 \mathrm{ng}$ DNA were subjected to amplification in reaction mixtures of $2.5 \mu \mathrm{l}$ (final volume

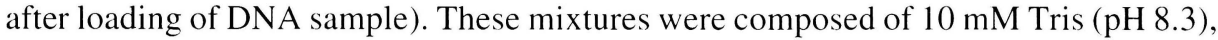
$50 \mathrm{mM} \mathrm{KCl}, 0.15 \mathrm{mg} / \mathrm{ml}$ gelatine, $0.1 \%$ Tween $20,200 \mu \mathrm{M}$ each dNTP, 0.0625 units Taq DNA polymerase (Roche Molecular Systems, Branchburg, N.J., USA), $1.75 \mathrm{mM} \mathrm{Mg}^{2+}$ (the primer pair $\mathrm{C} 1-\mathrm{C} 2$, however, were amplified in the presence of $2 \mathrm{mM} \mathrm{Mg2}{ }^{+}$), and 60 femtomol of upstream and downstream primer. For single strand conformation polymorphism (SSCP) analysis upstream as well as downstream primers were 5 ' end labelled with ${ }^{33} \mathrm{P}$. Amplification mixtures were prepared with unlabelled primers for subsequent cloning of amplification products. Samples were amplified through 35 cycles each consisting of $1 \mathrm{~min}$. at $94^{\circ} \mathrm{C}, 1 \mathrm{~min}$. at $55-60^{\circ} \mathrm{C}$ (annealing temperature depended upon primer pair) and $1 \mathrm{~min}$. at $72^{\circ} \mathrm{C}$. In the first cycle, however, denaturation at $94^{\circ} \mathrm{C}$ was prolonged by $45 \mathrm{sec}$. and in the last cycle, elongation $\left(72^{\circ} \mathrm{C}\right)$ was carried for a period of 6 mins. As an exception to this protocol, the primer pair D1-D3 was elongated at $72^{\circ} \mathrm{C}$ for just $15 \mathrm{sec}$. (this primer pair amplifies a fragment shorter than the others).

For SSCP analysis, the PCR products $(2.5 \mu \mathrm{l})$ were mixed with $5 \mu \mathrm{l}$ of formamide dye ( $95 \%$ formamide, $10 \mathrm{mM} \mathrm{Na}_{2}$ EDTA, $0.025 \%$ bromphenolblue and $0.025 \%$ xylolblue). After heating at $88^{\circ} \mathrm{C}$ for 5 min., 1-2 $\mu$ l of the denatured PCR products were subjected to SSCP analysis (Mashiyama et al., 1990).

Amplified fragments thought to represent different genetic variants, as judged by their SSCP migration pattern, were cloned for subsequent sequencing. Also a fragment encompassing the entire 5' LTR (amplified by the primer pair A1-D2) was cloned for 


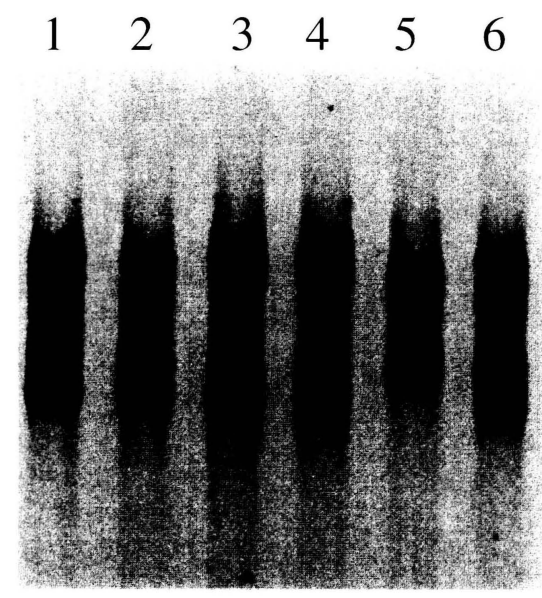

Figure 1. PCR-SSCP analysis using the B1-B2 primer pair. Note the appearance of two different homozygous patterns (lane 3 and 4 vs. lane 5) and a heterozygous pattern (lane 1,2 and 6).

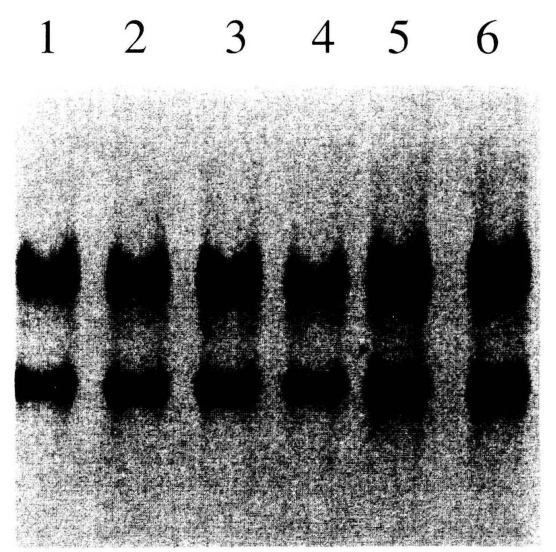

Figure 2. PCR-SSCP analysis using the C1-C2 primer pair. Polymorphic band patterns were not detected.

sequencing purposes. Briefly, the PCR products were blunt-ended and kinased after which they were inserted into a pUC 18 vector using the SureClone ${ }^{\mathrm{TM}}$ Ligation Kit, Pharmacia P-L Biochemicals Inc., Sweden. For the transfections we applied Escherichia coli $\mathrm{DH} 5 \alpha^{\mathrm{TM}}$ competent cells (Life Technologies, Gaithersburg, USA). Isolation of plasmids from bacterial clones relied upon a plasmid purification kit from Qiagen, Inc., Chatsworth, USA. Sequence determinations of the inserts were carried out using the Sequenase Version 2.0 USB ${ }^{\mathrm{TM}}$ kit (United States Biochemical Corporation, Cleveland, USA). Both strands were sequenced. For sequencing of the large amplified fragment, the standard M13 primers were supplemented with primers annealing to stretches in the insert (primer walking). To verify sequence data, PCR products were subjected to restriction fragment analyses using MseI, HindIII and Eco57I. 


\section{$\begin{array}{llllll}1 & 2 & 3 & 4 & 5 & 6\end{array}$}

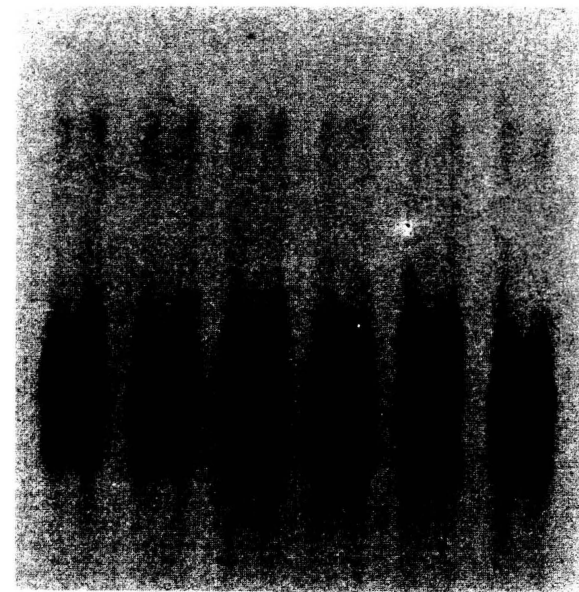

Figure 3. PCR-SSCP analysis using the D1-D2 primer pair. Note the appearance of a homozygous pattern (lane 1,2,3,4 and 6) and a heterozygous pattern (lane 5).

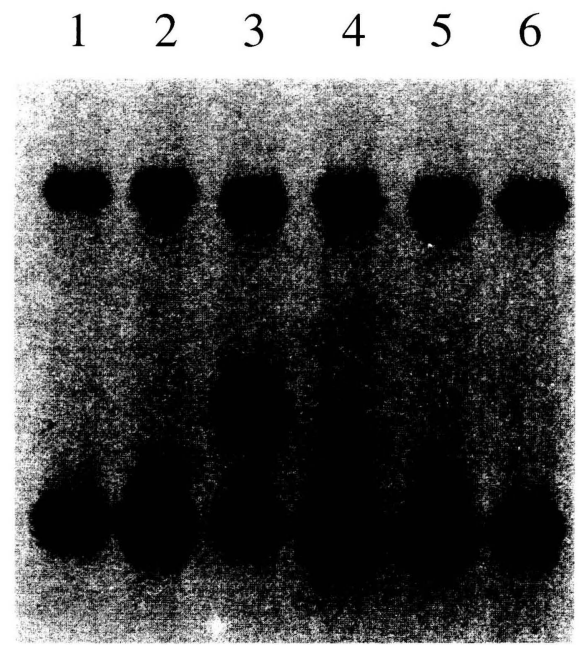

Figure 4. PCR-SSCP analysis using the D1-D3 primer pair. Note the appearance of a homozygous pattern (lane 1, 2, 4, 5 and 6) and a heterozygous pattern (lane 3).

A computer search was carried out to detect homologues of the $21 \mathrm{bp}$ imperfect repeat (AAGGCTCTGACGTCTCCCCCT), characteristic of the LTRs of HTLV-I and HTLVII (Shimotohno et al., 1986). The expected genotypic frequencies in a three allelic system under conditions of Hardy-Weinberg equilibrium were calculated using the trinomial $(p+q+r)^{2}$ where $p, q$ and $r$ represent the allelic frequencies. Subsequently, agreement between the expected and observed genotypic frequencies was examined by means of Chi-square test. 


\section{RESULTS}

All primer pairs produced fragments of the predicted sizes as determined by agarose gel electrophoresis. The primer pair $\mathrm{C} 1-\mathrm{C} 2$ also produced additional fragments of larger sizes. Moreover this primer did not amplify consistently. On SSCP analysis of samples amplified by the primer pair B1-B2, D1-D2 and D1-D3 polymorphic band patterns appeared (Figure 1, 3 and 4). This was not the case with fragments amplified by means of the primer pairs A1-A2 (not shown) and C1-C2 (Figure 2).

A continuous stretch of 918 nucleotides from three individuals (disregarding the sequences of the A1 and D2 primers) was sequenced. This stretch of nucleotides was compared with the previously published sequence of HRES-1 (Figure 5). The polymorphic band patterns observed on amplification with the B1-B2 primer pair reflected a $G$ $\rightarrow \mathrm{C}$ transversion of nucleotide number 437 creating a HindIII site. Sequencing of inserts produced by the D1-D2 primer pair, revealed a $G \rightarrow A$ transition at position 740 . This results in the disappearance of an Eco57I site, CTGAAG (N) $16^{\downarrow}$. Fragments amplified by the D1-D3 primer pair did not contain other polymorphisms besides that involving nucleotide number 740 . Sequencing of the A-rich stretch produced varying results in that nine clones contained 11 A's in contiguity whereas 12 A's were observed in three clones. Using MseI for restriction of the product amplified by means of the B1-B2 primer pair, fragments of about 175, 80 and $50 \mathrm{bp}$ were recorded (not shown). On the basis of our sequence data, detection of three restriction fragments of about 169, 76 and 54 bp was expected, whereas the previously published sequence predicts that $M s e$ I treatment of the amplification product in question generates two fragments of 125 and $166 \mathrm{bp}$. Finally, the presence of a HindIII and an Eco57I polymorphic site in HRES-1 was confirmed (not shown). Elements corresponding to the 21 nucleotide repeat of HTLV were not detected in the LTR of HRES-1. The individuals were arranged according to the genotypes determined by the HindIII marker and the Eco57I marker, respectively (Table 2). The Eco57I negative allele was only detected in individuals lacking the HindIII polymorphic site on both chromosomes ( 12 individuals) and in HindIII heterozygotes ( 6 individuals). The relationship between the two markers allowed us to define three HRES-1 alleles, namely HRES- $1^{1}$, HRES- $1^{2}$ and HRES- ${ }^{3}$ (Figure 6). Using this nomenclature, we have listed the genotypic frequencies and the frequencies of the alleles (Table 3). The genotypic frequencies did not differ significantly from the values expected under conditions of Hardy-Weinberg equilibrium (calculations not shown).

\section{DISCUSSION}

Our HRES-1 sequence differs from the previously published data (Perl et al., 1989) in various aspects. Whether the disagreements between our sequence data and the previously published HRES-1 sequence reflect a different genetic background is uncertain. The possibility that we have amplified fragments from different copies of proviral elements closely related to HRES-1 seems to be less likely since we sequenced a considerable number of clones with consistent results, including clones with an insert containing the entire HRES1 LTR (about $1 \mathrm{~kb}$ ). As HRES-1 lacks a downstream LTR the possibility can be excluded that we amplified fragments from such HRES-1 region. The varying sequencing results we recorded in the A-rich region probably reflect an inability of the Taq DNA polymerase to copy this stretch in a precise manner. The finding that some of the sequence variations between the 


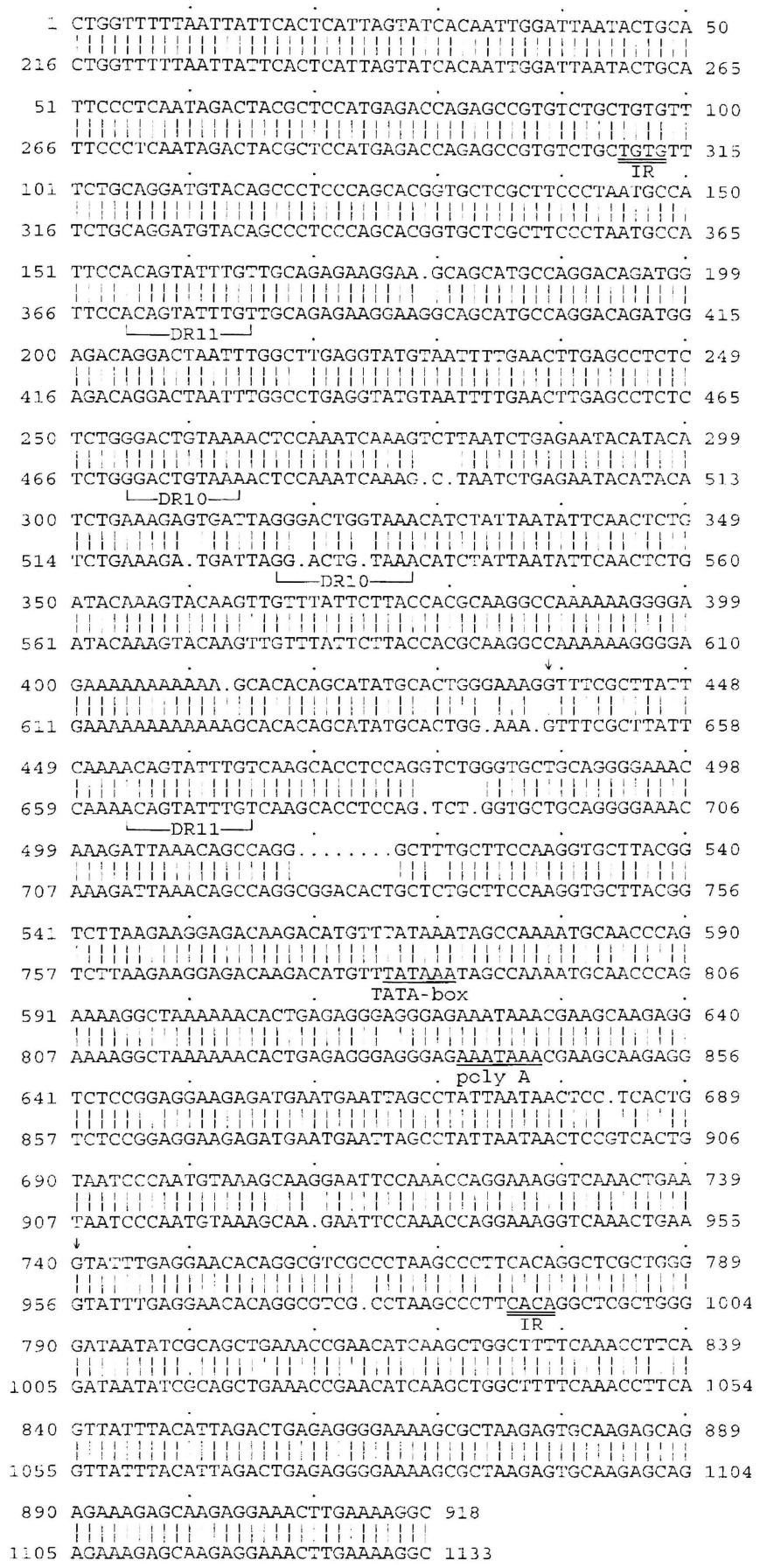

Figure 5. Comparison of sequences of the upstream portion of HRES-1. The upper sequence represents data from the present study; the lower one is the previously published HRES-1 sequence (Perl et al., 1989). Inverted repeats (IR), direct repeats (DR), a TATA box and a polyadenylation signal (poly A) are indicated. Arrows pin-point the polymorphic nucleotides. 


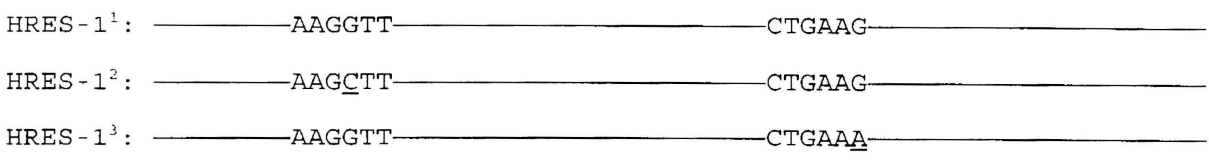

Figure 6. Three alleles of HRES- 1 . HRES- $1^{2}$ is distinguished from HRES- $1^{1}$ by having a $\mathrm{G} \rightarrow \mathrm{C}$ transversion at nucleotide number 437 , creating a Hind III site (A $\downarrow$ AGCTT); HRES- $1^{3}$ is distinguished from HRES- $1^{1}$ by a $\mathrm{G} \rightarrow \mathrm{A}$ transition of nucleotide number 740 . This abolishes an Eco57I recognition site (CTGAAG (N) $\left.16{ }^{\downarrow}\right)$. The two nucleotides distinguishing HRES- $1^{2}$ and HRES $-1^{3}$ from HRES- $1^{1}$ are underlined.

Table 2. Relationship between the HindIII and Eco57I polymorphisms of HRES-1a.

\begin{tabular}{lccrrr}
\hline & & HindIII type: & $-/-$ & $+/-$ & $+/+$ \\
\hline \multirow{2}{*}{ Eco57I } & type: & $+/+$ & 68 & 56 & 16 \\
& $+/-$ & 12 & 6 & 0 \\
\hline
\end{tabular}

a. Number of individuals in the groups: $-/-$ (homozygotes lacking the restriction site),$+/+$ (homozygotes carrying the restriction site) and $+/$ - (heterozygotes).

Table 3. Distribution of genotypes and alleles of HRES-1.

\section{Number of individuals ${ }^{\mathrm{a}}$}

\section{Genotypes}

HRES- $1^{1} / \mathrm{HRES}-1^{1}$

HRES- 1 / HRES- 12

HRES-12/HRES- 12

HRES- 1 / HRES- 13

HRES- $12 /$ HRES- 13

HRES- $13 /$ HRES- 13

Alleles

HRES- 1

HRES- 12

HRES- 13

a. Frequencies are listed in brackets.
$68(0.43)$

$56(0.35)$

$16(0.10)$

$12(0.08)$

$6(0.04)$

$0(0.00)$

Number of chromosomesa

previously published sequence and ours involved regions used for design of primers could explain the inconsistent results obtained with one of the primer pairs.

Elements characteristic of retroviral LTRs were reported in the previous HRES-1 sequence (Perl et al., 1989), including indirect repeats, a TATA box, a polyadenylation signal and two direct repeats (DR) spanning 10 nucleotides and 11 nucleotides, respectively. Apart from the 10 nucleotide DR, these elements were also found in our sequence. 
The LTR of HRES- 1 also contains stretches of homology with HTVL-I and HIV-2 (Perl et al., 1989) such as a short stretch (CTCTCTGG) of the TAR element of HIV (Selby et al., 1989). So far, the significance of this stretch is unclear, especially, in view of its localization several hundreds of nucleotides upstream of the promotor. The absence of the 21 nucleotide imperfect repeats characteristic of HTLV LTR points towards a more distant relationship between HRES-1 and HTLV. Previously, a polymorphic HindIII site was reported in HRES-1 (Perl et al., 1989). Most probably, this site corresponds to our point mutation at position 437. The linkage between the HindIII and Eco57I markers in the LTR of HRES-1 permitted us to define three alleles of this endogenous retrovirus. Whether the coding regions of HRES- 1 also are subject to genetic variation is not known yet.

The establishment of HRES-1 alleles permits future studies of possible associations between this endogenous retrovirus and various diseases. In a subsequent study we have detected a significant difference in the distribution of HRES- 1 alleles between multiple sclerosis patients and healthy individuals (H.B. Rasmussen et al., submitted).

\section{ACKNOWLEDGEMENTS}

Lisbeth Jørgensen is thanked for technical assistance. This work is part of the retrovirus research program of the Danish Multiple Sclerosis Society.

\section{REFERENCES}

Brodsky, I., Foley, B., Haines, D., Johnston, J., Cuddy, K., Gillespie, D. (1993). Expression of HERV-K proviruses in human leukocytes. Blood, 81, 2369-2374.

Larsson, E., Kato, N., Cohen, M. (1989). Human endogenous proviruses. In: Vogt, P.K. (Ed.). Oncogenes and retroviruses. Curr. Topics Microbiol. Immunol., 148, 115-132.

Mashiyama, S., Sekiya, T., Hayashi, K. (1990). Screening of multiple DNA samples for detection of sequence changes. Technique, 2, 304-306.

Mountz, J.D., Talal, N. (1993). Retroviruses, apoptosis and autogenes. Immunol. Today, 14, 532536.

Perl, A., Isaacs, C.M., Eddy R.L., Byers, M.G., Sait, S.N.J., Shows T.B. (1991). The human T-cell leukemia virus-related endogenous sequence (HRES1) is located on chromosome 1 at q42. Genomics 11, 1172-1173.

Perl, A., Rosenblatt, J.D., Chen, I.S.Y., DiVincenzo, J.P., Bever, R., Poiesz, B.J., Abraham, G.N. (1989). Detection and cloning of new HTLV-related endogenous sequences in man. Nucleic Acids Res., 17, 6841-6854.

Sauter, M., Schommer, S., Kremmer, E. Remberger, K., Dölken, G., Lemm, I., Buck, M., Best, B., Neumann-Haefelin, D., Mueller-Lantzsch, N. (1995). Human endogenous retrovirus K10: expression of gag protein and detection of antibodies in patients with seminomas. J. Virol., 69, 414-421.

Selby, M.J., Bain, E.S., Luciw, P.A., Peterlin, B.M. (1989). Structure, sequence, and position of the stem-loop in tar determine transcriptional elongation by tat through the HIV-1 long terminal repeat. Genes Dev., 3, 547-558.

Shimotohno, K., Takano, M., Teruuchi,T., Miwa, M. (1986). Requirement of multiple copies of a 21-nucleotide sequence in the $U 3$ regions of human T-cell leukemia virus type I and type II long terminal repeats for trans-acting activation of transcription. Proc. Natl. Acad. Sci. USA, 83, 8112-8116.

Sutherland, G.R., Parslow, M.I., Baker, E. (1985). New classes of common fragile sites induced 
by 5-azacytidine and bromodeoxyuridine. Hum. Genet., 69, 233-237.

Whartenby, K.A., Insel, R.A., Freeman, S.M., Keng, P., Abraham, G.N. (1993). Oncogene elements within an endogenous retrovirus. Acta Virol., 37, 113-122. 


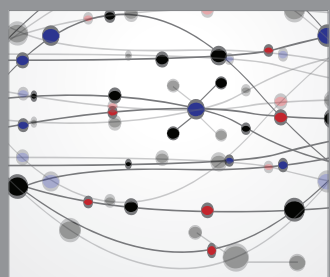

The Scientific World Journal
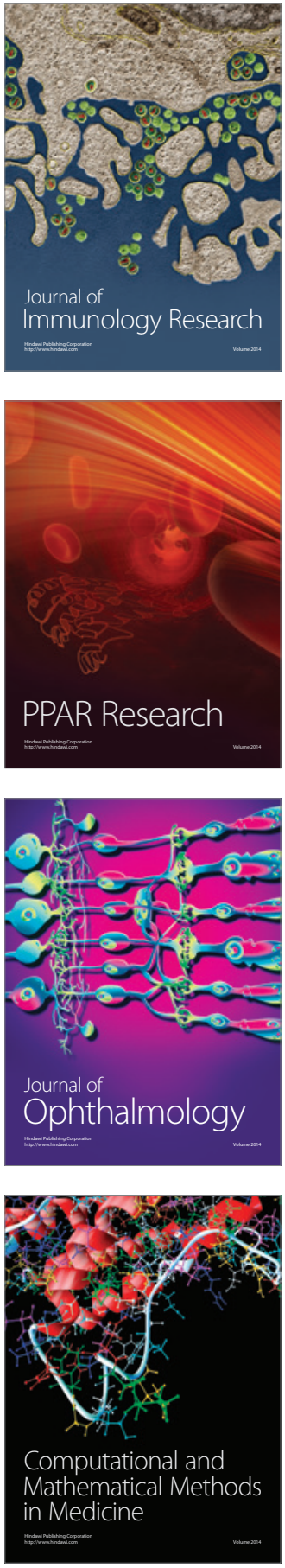

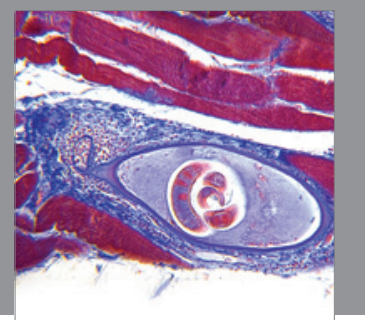

Gastroenterology

Research and Practice
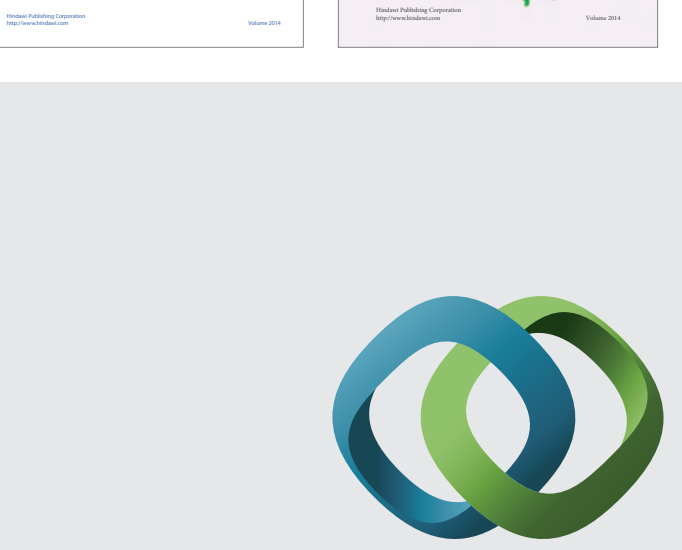

\section{Hindawi}

Submit your manuscripts at

http://www.hindawi.com
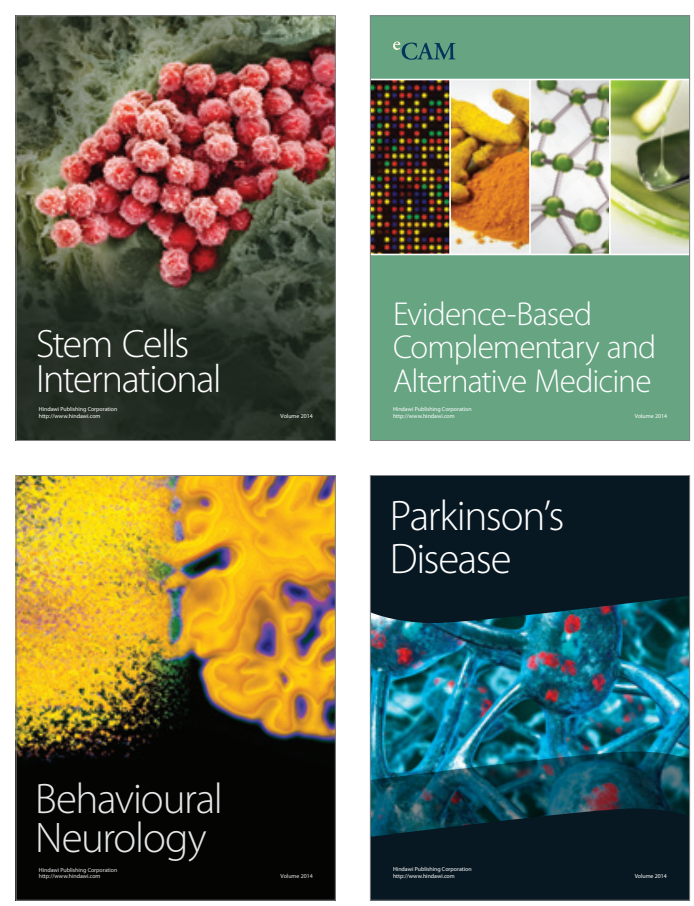

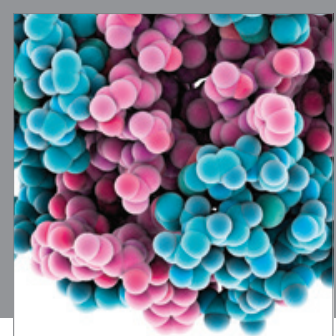

Journal of
Diabetes Research

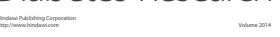

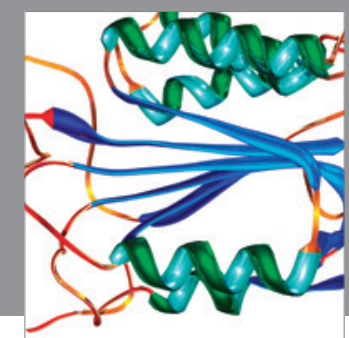

Disease Markers
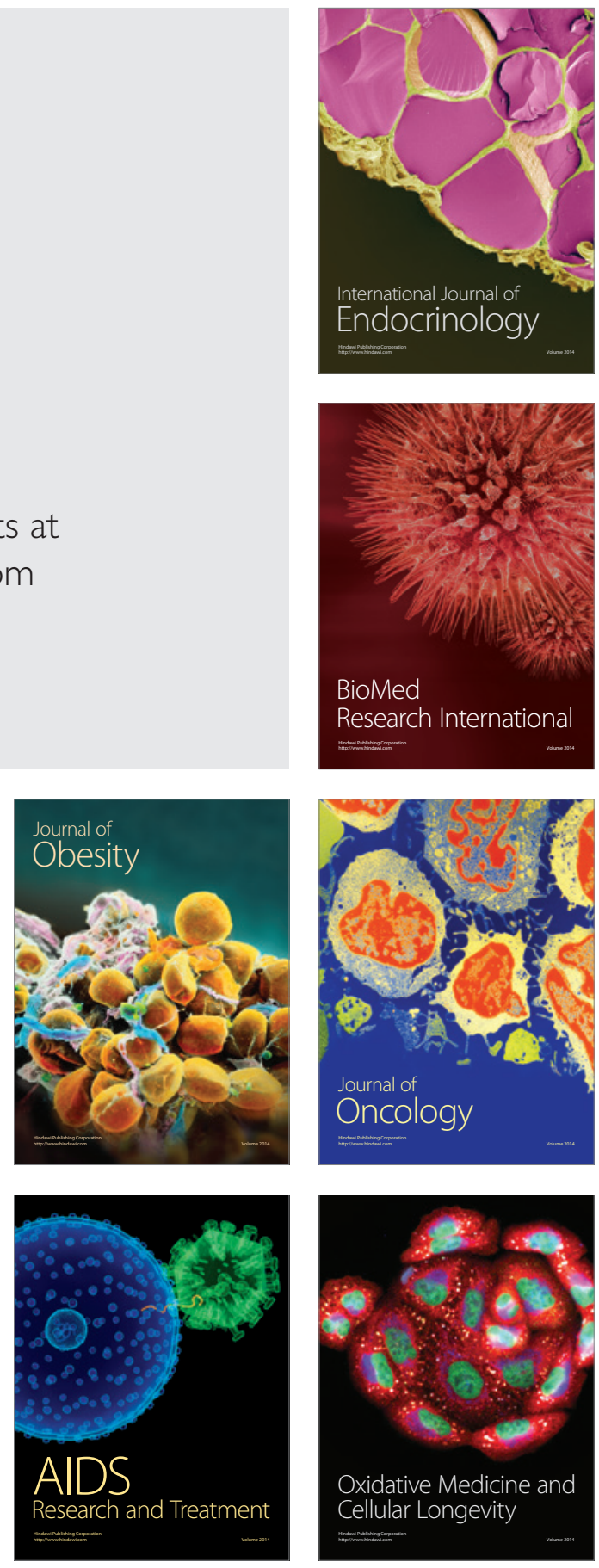